\title{
Estimating the size of myocardial infarction by magnetic resonance imaging
}

\author{
L W Turnbull, J P Ridgway, J J Nicoll, D Bell, J J K Best, A L Muir
}

\begin{abstract}
Objective-To develop a method to measure myocardial infarct size by magnetic resonance imaging and to compare the results with pyrophosphate scanning by single photon emission computed tomography.

Design-All patients underwent magnetic resonance imaging and pyrophosphate scanning 5-7 days after the onset of symptoms. Both measurements of infarct size were compared with the release of creatine kinase $M B$ and with ventricular performance estimated by radionuclide ventriculography.
\end{abstract}

Patients-19 patients (age 40-68 years) who had sustained their first uncomplicated myocardial infarction and who had not been treated with thrombolytic therapy.

Results-The site of infarction was clearly shown by both imaging techniques and was identical in each patient. The volume of infarcted tissue measured by magnetic resonance imaging agreed well with the infarct size measured by single photon emission tomography (mean difference $2 \cdot 7 \mathrm{~cm}^{3}$ ). Correlations of both imaging techniques with the release of creatine kinase $M B$ were best when total release rather than peak release was used. Both imaging techniques correlated closely with the subsequent ventricular performance.

Conclusions-Magnetic resonance imaging after acute infarction allows measurement of infarct size and this may prove useful in assessing new treatments designed to salvage myocardium.

Prognosis after acute myocardial infarction depends on the extent of myocardial damage and on residual left ventricular function. Salvage of acutely ischaemic but reversibly damaged myocardium is widely accepted to be responsible for the preservation of ventricular function and for improved survival after thrombolytic therapy. ${ }^{12}$ However, there are discrepancies between survival after reperfusion and residual left ventricular function, ${ }^{3}$ and it has been suggested that the beneficial effects of thrombolysis may be more complex than a simple reduction in infarct size. Currently, ventricular function is used as an indirect indicator of the mass of ventricle infarcted, but in patients with previous infarction this relation is distorted. More direct measurements of infarct size are required to clarify the effects of intervention.

Of the other standard techniques (including ST segment and $R$ wave mapping, cardiac enzyme release, and echocardiography) only radionuclide imaging with infarct avid agents attempts directly to measure the mass of damaged myocardium. Recent reports suggest that magnetic resonance imaging may offer considerable advantages for direct measurement of infarct size. ${ }^{45}$ Studies in both animals and humans have shown that infarcted muscle can be recognised on gated magnetic resonance images and we showed that there is an increase in the relaxation parameter, $\mathrm{T} 1$, in patients after acute infarction. ${ }^{6}$ In animal studies there was a close relation between infarct size measured from areas of increased pixel intensity on magnetic resonance images and anatomical estimates of myocardial injury identified histochemically. ${ }^{?}$

To extend these observations to humans we examined myocardial infarct size with a low field magnetic resonance imaging system and compared the estimated injury with single photon emission tomographic images of $99 \mathrm{~m}$ technetium pyrophosphate uptake, with release of the enzyme creatine kinase $M B$, and with residual left ventricular function measured by radionuclide ventriculography.

\section{Patients and methods}

PATIENTS

We studied 19 patients (14 men and five women) who had sustained their first myocardial infarction and who had not been treated with thrombolytic drugs. Myocardial infarction was diagnosed on the basis of history and typical electrocardiographic or cardiac enzyme changes or both. At the time of the examination all patients were clinically stable with no evidence of either acute pulmonary oedema or low output cardiogenic shock. The patients were all in sinus rhythm and gave informed consent before entering the study. None of the patients gave a history of previous cardiac disease or of other chronic medical conditions.

\section{MAGNETIC RESONANCE IMAGING}

Technique

In all patients magnetic resonance imaging was carried out five to seven days after the onset of chest pain with a low field system (M \& D Technology) operating at $0.08 \mathrm{~T}$. Patients with either temporary or permanent cardiac pacemakers, prosthetic heart valves, or previous intracranial surgery had already been 
Figure 1 (A) T1 map of an angled transverse image obtained through the ventricles showing a large semicircular area of reduced signal intensity involving the interventricular septum and adjacent anterior left ventricular wall secondary to infarction. (B) Areas within the myocardium with a $T 1$ value two standard deviations or more above the mean $T 1$ value for normal myocardium are shown in white.
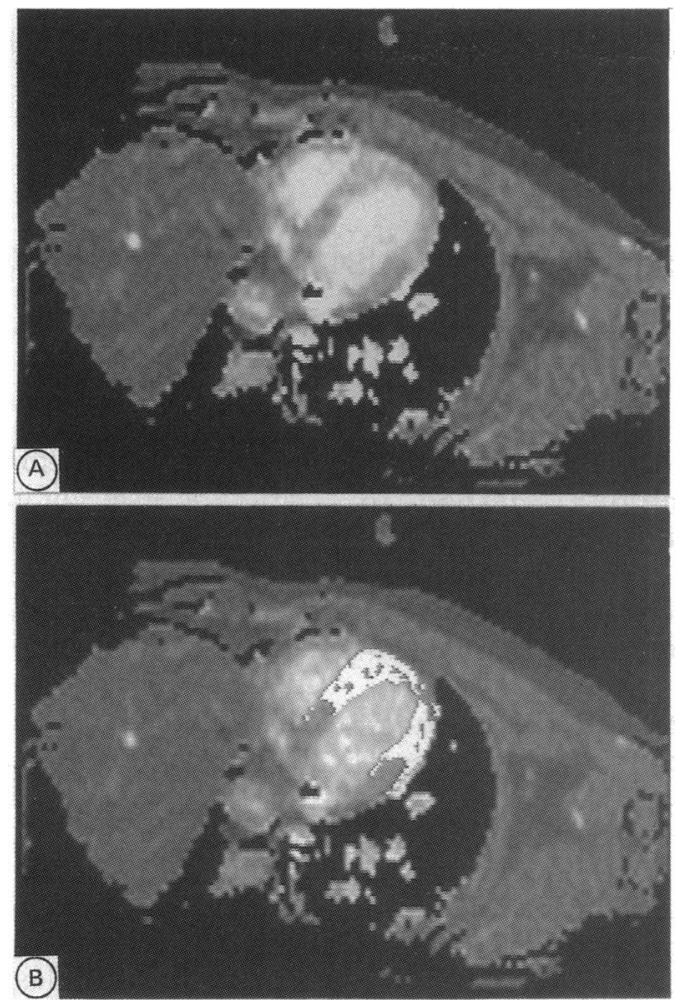

excluded from the study. Scout images were initially carried out in the coronal plane to visualise the long axis of the ventricles. These images were obtained with a field of view of $384 \mathrm{~mm}$, a matrix of $128 \times 64$, a short repetition time (TR) of $240 \mathrm{~ms}$, and a field echo time of $22 \mathrm{~ms}$. Four slices were obtained with a $16 \mathrm{~mm}$ slice thickness and separation. From these images, short axis views of the ventricular chambers were aligned. We used a cardiac gating technique with carbon fibre electrodes (Abingdon Instruments, Abingdon, Oxford) placed on the left anterolateral and the right parasternal chest wall. ${ }^{8}$ The downslope of the electrocardiographic $\mathrm{T}$ wave was used to synchronise data acquisition during end systole at approximately $110 \mathrm{~ms}$ after the upstroke of the $T$ wave. An interleaved saturation recovery-inversion recovery pulse sequence was used with a TI of $42 \mathrm{~ms}$ and a variable $T R$, depending on the RR interval. Eight to 10 slices (12 mm thick) were acquired as four acquisitions to obtain multiple images from below the cardiac apex to the level of the aortic arch. Two slices with a $42 \mathrm{~ms}$ time separation were obtained with each acquisition which consisted of two averages of 64 phase encoding steps and 128 frequency encoding steps resulting in a pixel size of $3 \times 6 \mathrm{~mm}$. These data were interpolated to a $128 \times 128$ matrix $(3 \times 3 \mathrm{~mm})$ and smoothed on the final display monitor for image analysis.

\section{Analysis}

The calculated T1 map showed good contrast between normal myocardium, ischaemic myocardium, and chamber contents, and was used to define the myocardial border (fig 1A). The outlines of the endocardial and epicardial interfaces of the left ventricle, including the interventricular septum, were defined interactively by using image analysis software to draw an irregular region of interest. Owing to partial volume effects from pericardial fat the $T 1$ values of the extreme apical segment were frequently higher than those of the normal myocardium, and these images were therefore excluded from analysis. The T1 calculations ${ }^{9}$ were based on a look-up table that was generated from the saturation recovery and "difference" image signals when a repetition time of $1000 \mathrm{~ms}$ was assumed. However, because it was shown from phantom experiments that our measurements of $T 1$ values, which are based on these look-up tables, varied considerably with heart rate it was necessary to calculate an upper limit of normal for each individual patient. This was done by taking the mean $\mathrm{T} 1$ value plus two standard deviations from regions of a fixed size (approximately $2 \mathrm{~cm}^{3}$ ) placed within normal myocardium on all the slices that included the left ventricle. These regions of interest were as remote as possible from the areas of increased signal and were retrospectively confirmed to be separate from regions of increased pyrophosphate uptake. An average T1 value was obtained for all the slices measured. Regions within the myocardium with a $T 1$ value greater than this limit were then generated by the computer (fig 1B) and their areas measured. These values were multiplied by the slice thickness to obtain the volume of the infarcted myocardium on each slice, and then these volumes were summed to obtain the total volume of infarcted myocardium.

\section{REPRODUCIBILITY}

The reproducibility of the technique was affected by the measurement of both the mean and standard deviation of normal myocardium and by identification of the endocardial and epicardial borders of the left ventricle. To determine the intra and inter observer variability two independent observers performed measurements on the same four patient examinations on three separate occasions.

The volume of infarcted myocardial tissue varied by $10.1 \%$ between observers and the intra observer variability was 6.8 and $10.4 \%$. This variation was primarily attributable to the difference of $8.8 \%$ between the two measurements of the upper threshold limit for normal myocardium (mean (2 SD)).

99M-TECHNETIUM PYROPHOSPHATE SCANNING Radioisotope scanning with $99 \mathrm{~m}$-technetium pyrophosphate was performed with a MaxiCamera 400AT Gamma Camera equipped with a low energy, general purpose, parallel hole collimator (International General Electric) and a MicroDelta Plus computer system (Siemens Gammasonics). Patients were imaged between 24 and 36 hours after admission to hospital and two hours after administration of $600 \mathrm{MBq} 99 \mathrm{~m}$-technetium pyrophosphate. Patients were positioned supine with both arms beside the head. Single photon emission tomography was performed with a set of sixty four, $64 \times 64$ images taken 
over a $360^{\circ}$ arc with the radius of rotation selected to minimise the distance from the detector to the chest wall. Each image was collected for a preset time of 10 seconds and, in addition to the "auto-tune" facility, the camera was further corrected for uniformity (using a 30 million count ${ }^{99 \mathrm{~m}} \mathrm{Tc}$ flood) and for the centre of rotation. Transverse images, one pixel thick, were reconstructed "on line" with a Butterworth filter of order 5 and a cut-off frequency of 0.5 Nyquist. The myocardial uptake of pyrophosphate was determined from the transverse images by a simple three dimensional thresholding procedure operating at $65 \%$ of the peak voxel value within the infarct volume. The threshold value of $65 \%$ has been shown ${ }^{10}$ from phantom studies to be the optimal value. The peak value in the volume of interest was found after each transverse slice had been smoothed twice by a nine point filter. Myocardial uptake was analysed by drawing a cuboid region of interest around the heart and counting the number of voxels $>65 \%$ of the peak value within that volume (fig 2). The number of infarct voxels was then summed and the size of infarction determined by multiplying by the voxel size $\left(0.24 \mathrm{~cm}^{3} /\right.$ voxel $)$.

Before we calculated infarct volume we reviewed each transverse image, and if necessary, modified the region of interest to exclude areas of non-cardiac uptake such as the sternum or ribs.

\section{SERUM CREATINE KINASE MB}

Serum creatine kinase $M B$ was measured by an optimised kinetic spectrophotometer assay. In 11 of the 19 patients, 4-8 blood samples were obtained from eight to 48 hours after the onset of symptoms. This allowed calculation of the total creatine kinase released. In the remaining eight patients only three blood

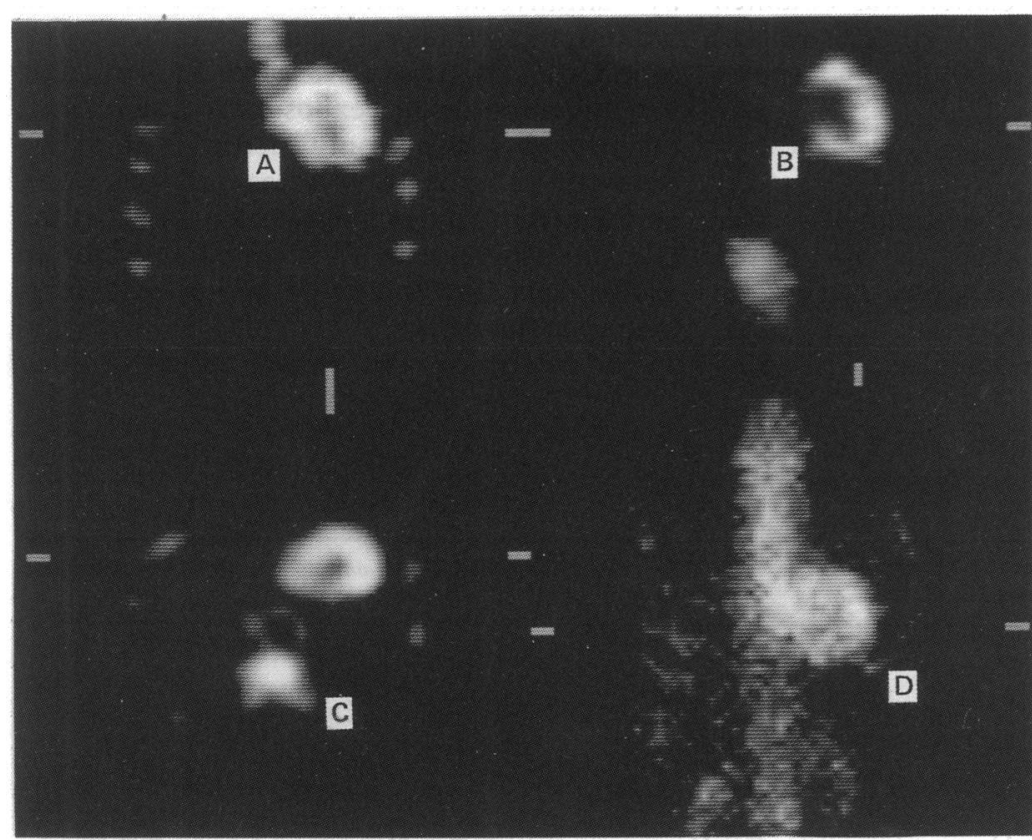

Figure 2 Single photon emission tomographic images of ${ }^{99 m} T c$-pyrophosphate uptake in the myocardium of the same patient as fig $1 . A$ and $B$ show an extensive anterior infarction. (A) coronal section; (B) sagittal section; (C) transverse section; (D) scout image roughly equating to a planar anterior view. samples were obtained and we used the highest value to express a "peak" creatine kinase release. Both the total and the peak creatine kinase release were used in our correlations.

\section{RADIONUCLIDE VENTRICULOGRAPHY}

This was carried out on the day before discharge from hospital (mean 7 days), when the patient was clinically and radiologically clear of heart failure. All the patients were studied in the supine position and cardiac imaging was performed in approximately a $30^{\circ}$ left anterior oblique projection with a $10^{\circ}$ caudal tilt. The precise angle was chosen to optimise the separation of the ventricles. After injection of $700 \mathrm{MBq}{ }^{99 m} \mathrm{Tc}$ human serum albumin, imaging was carried out with a Siemens low energy mobile gamma camera and the data were collected by a Micro Delta computer. The ejection fraction was calculated by a gated blood pool technique. ${ }^{11}$

STATISTICAL ANALYSIS

Statistical analysis was performed with the SPSS/PC + computer programme.

\section{Results}

The location of the infarct was clearly demonstrated and was identical by both pyrophosphate scanning and magnetic resonance imaging in 17 of the 19 patients. No abnormality was shown by either imaging technique in one patient, while in a second pyrophosphate imaging showed only a very small area of increased uptake anteriorly, but magnetic resonance imaging failed to demonstrate any abnormality. In the first of these two patients the electrocardiogram was normal and in the second it was indeterminate owing to the presence of left bundle branch block. Both patients showed only a minor rise in the peak serum creatine kinase $M B$ values of 172 and $522 \mathrm{U} / 1$ respectively.

We compared the myocardial infarct size measured by pyrophosphate scanning and magnetic resonance imaging by plotting the difference between the two values against the mean infarct size obtained from both methods ${ }^{12}$ (fig 3). This showed a mean difference of $2.7 \mathrm{~cm}^{3}$ (magnetic resonance infarct size minus pyrophosphate infarct size), while the standard deviation of the difference was $9.9 \mathrm{~cm}^{3}$. The limits of agreement were -22.5 and $17.1 \mathrm{~cm}^{3}$ and the standard error of the mean was $2.27 \mathrm{~cm}^{3}$. Hence the $95 \%$ confidence interval for the lower limit of agreement was $-30 \cdot 8$ to $-14.3 \mathrm{~cm}^{3}$, while for the upper limit of agreement the $95 \%$ confidence interval was 8.9 to $25.4 \mathrm{~cm}^{3}$. These intervals showed moderately good agreement between the two methods, with reasonable consistency over the range of values encountered, although discrepancies were noted with infarct sizes $>80 \mathrm{~cm}^{3}$.

The table shows the correlations between infarct size, creatine kinase $M B$ release, and left ventricular ejection fraction. The creatine kinase $\mathrm{MB}$ release curves could be calculated in only 11 patients, but there was a good correlation between the peak and total creatine kinase 


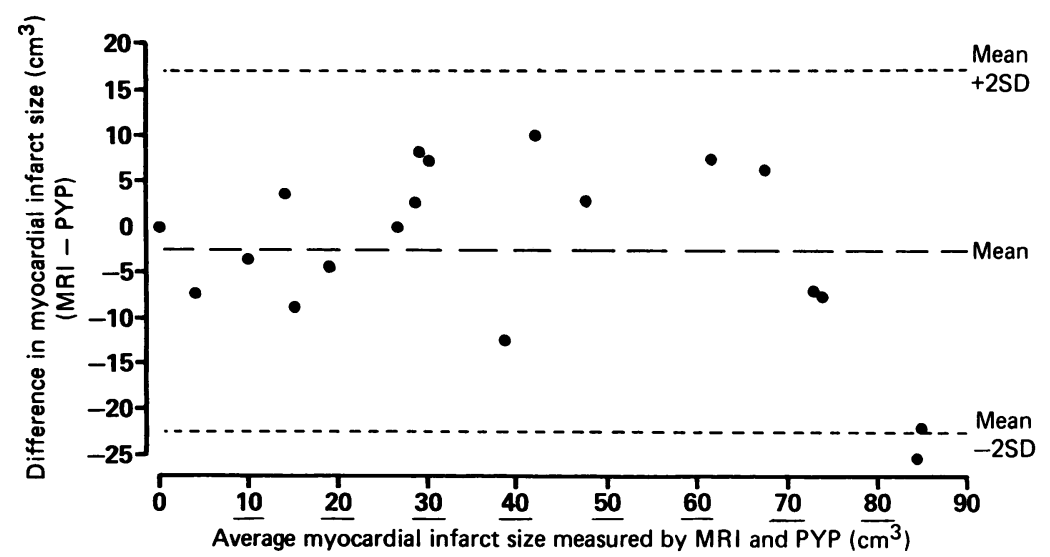

Figure 3 Difference in myocardial infarct size between magnetic resonance imaging (MRI) and ${ }^{9 m}$ Tc-pyrophosphate scanning (PYP) plotted against the average value measured by these techniques.

values. The size of the infarct measured by peak creatine kinase correlated only moderately with the volume of the infarct measured by pyrophosphate $(r=0.53,0.02>p>0.01)$ and by magnetic resonance imaging $(\mathrm{r}=0.52,0.05>$ $\mathrm{p}>0.02$ ). The correlations were weakened by the presence of two particularly high creatine kinase results (6955 and 6255) that were considerably greater than the other values. However, the total creatine kinase release, which included these two values, correlated well with the infarct size measured by both pyrophosphate $(r=0.75,0.01>p>0.001)$ and magnetic resonance imaging $(r=0.62$, $0.05>p>0.02)$. The residual left ventricular function measured by radionuclide ventriculography correlated significantly with the volume of infarct measured by both magnetic resonance $(r=0.79, p<0.001)$ and pyrophosphate imaging $(\mathrm{r}=-0.79, \mathrm{p}<0.001)$. There was also a good correlation between the left ventricular ejection fraction and the peak creatine kinase release $(r=-0.63,0.01>p>$ 0.001 ), but a better correlation was obtained with the total creatine kinase release $(\mathrm{r}=-0.78,0.01>\mathrm{p}>0.001)$.

\section{Discussion}

The primary determinant of prognosis after acute myocardial infarction is the size of the area of infarction. ${ }^{13-15}$ However, despite its importance, routine clinical measurement of infarct size has not been achieved. The tech- niques currently used are indirect and may be misleading after thrombolytic therapy. Peak serum creatine kinase values are more important. Analysis of creatine kinase MB release curves correlates well with histological infarct size and provides prognostic information. ${ }^{16-18}$ However, after thrombolytic therapy there is an early accelerated "washout" of cardiac enzymes, presumably when blood flow is restored through partially necrotic myocardium. ${ }^{19}$ Electrocardiographic techniques are used to map the extent of infarction but are inaccurate in assessing small non-transmural or posterior lesions. ${ }^{2021}$ Echocardiography is mainly of use in determining areas of wall thinning, dyskinesis, or akinesis, and hence also has major limitations. Residual ventricular function gives an indication of myocardial damage but is unreliable when there has been previous infarction. Tissue characterisation by any imaging technique remains an elusive goal.

Since the introduction of single photon emission tomography, pyrophosphate imaging has been considered a sensitive method for the diagnosis and measurement of acute myocardial infarcts. ${ }^{22}$ The use of single photon emission tomography has allowed more accurate detection of posterior, inferior, and small subendocardial infarcts, particularly after the addition of blood pool overlay. ${ }^{23}$ These data correlated well with the histological findings in animals, ${ }^{23}$ and with analysis of serum creatine kinase $\mathrm{MB}$ release and residual left ventricular function in humans.

There are certain limitations to pyrophosphate imaging. The delivery of the radionuclide to the centre of a large infarct may be incomplete. ${ }^{24}$ Our single photon emission tomographic technique overcomes this by measuring the volume defined by pyrophosphate rather than by quantifying the amount of isotope concentrated in the unevenly perfused tissue. More uniform labelling can be achieved by antimyosin antibodies, labelled with indium, but the poorer imaging characteristics of indium offset this advantage. Radionuclide imaging also involves exposure to a significant radiation dose and is only useful for up to five days after infarction, whereas our own work, performed in human subjects, showed that the maximum increase in $\mathrm{T} 1$ is not obtained for up to five days after infarction and persists for a long time thereafter. ${ }^{25}$

To determine the extent of myocardial

Correlations between measurements of infarct size, left ventricular ejection fraction $(\%)$, and release of creatine kinase $M B(U \mid l)$

\begin{tabular}{lllll}
\hline & $\begin{array}{l}\text { MRI } \\
\text { infarct size }\end{array}$ & $\begin{array}{l}\text { 99m Tc PYP } \\
\text { infarct size }\end{array}$ & $\begin{array}{l}\text { LV ejection } \\
\text { fraction }\end{array}$ & $\begin{array}{l}\text { Peak creatine } \\
\text { kinase }\end{array}$ \\
\hline LV ejection fraction & $\mathrm{n}=18$ & $\mathrm{n}=18$ & \\
& $\mathrm{r}=-0.79$ & $\mathrm{r}=-0.79$ & \\
$\mathrm{p}<0.001$ & $\mathrm{p}<0.001$ & $\mathrm{n}=17$ \\
Peak creatine kinase & $\mathrm{n}=18$ & $\mathrm{n}=18$ & $\mathrm{r}=-0.63$ \\
& $\mathrm{r}=0.52$ & $\mathbf{r}=0.53$ & $0.01>\mathrm{p}>0.001$ & $\mathrm{n}=11$ \\
Total creatine kinase & $0.05>\mathrm{p}>0.02$ & $\mathrm{n}=02 \mathrm{p}>0.001$ & $\mathrm{n}=11$ \\
& $\mathrm{n}=11$ & $\mathrm{n}=11$ & $\mathrm{r}=0.93$ \\
& $\mathrm{r}=0.62$ & $\mathrm{r}=0.75$ & $0.01>\mathrm{p}>0.001$ & $\mathrm{p}<0.0001$ \\
\hline
\end{tabular}

LV, left ventricle; MRI, magnetic resonance imaging; PYP, pyrophosphate. 
infarction by magnetic resonance imaging, we had to define $\mathrm{T} 1$ values for normal myocardium. Because the measured $\mathrm{T} 1$ values obtained from our system varied with repetition time it was essential to define a threshold limit for each individual patient. Although normal myocardium was identified subjectively the inter observer variability of $10 \cdot 1 \%$ suggests that this is an acceptable method. In this study we related the volume of infarcted myocardium measured by magnetic resonance imaging five to seven days after infarction to several variables that also reflect infarct size.

We chose to obtain the magnetic resonance images at this stage because our previous work had shown this to be the time of maximal change. We studied patients with their first myocardial infarction so that preservation of ventricular function after infarction would negatively correlate with infarct size. In addition, because our patients were not treated with thrombolytic therapy the creatine kinase kinetics would be unaltered. Our results indicate that with a low field magnetic resonance imaging system T1 maps provide estimates of infarct size that agree closely with other techniques. We found a good agreement between magnetic resonance and pyrophosphate imaging and both these techniques correlated well with depressed ventricular function measured after infarction. Poorer correlations were obtained with peak creatine kinase but these were improved in the group of patients for whom enough data points were available to calculate the total release of creatine kinase MB.

We expect that the use of a $128 \times 128$ pixel matrix and a reduced slice thickness will improve the precision of infarct size measurement by magnetic resonance imaging although it will increase the examination time. Many factors, such as the field strength and the imaging pulse sequence, can alter the ability to detect myocardial damage. Currently the second or third echo of a multiple spin echo sequence is widely used to detect myocardial infarction, but particularly at high field strengths a paramagnetic contrast agent, such as gadolinium diethylenetriamine pentaacetic acid may then be required to demonstrate areas of damage. ${ }^{26}$ This study shows the ability of a low field system with a saturation recoveryinversion recovery plus sequence to measure human infarct size and avoid the routine use of paramagnetic contrast agents. Because T1 values remain raised for the first three months after infarction, ${ }^{25}$ this technique will provide information on infarct size in the convalescent period.

We thank Mrs R H B Armstrong and Miss F Taddei for their excellent technical help. This work was supported in part by the British Heart Foundation.

1 Gruppo Italiano per lo Studio della Streptochinasi nell' Infarto Miocardico (GISSI). Effectiveness of intravenous thrombolytic treatment in acute myocardial infarction Lancet 1986;i:397-401.
2 ISIS-2 (Second International Study of Infarct Survival) Collaborative Group. Randomised trial of intravenous streptokinase, oral aspirin, both, or neither among 17,187 cases of suspected acute myocardial infarction: ISIS-2. Lancet 1988;ii:349-60.

3 White HD, Norris RM, Brown MA, Takayama M, Maslowski A, Bass NM, et al. Effects of intravenous streptokinase on left ventricular function and early 317:855-8.

4 Rokey R, Verani MS, Bolli R, Kuo LC, Ford JJ, Wendt RE, et al. Myocardial infarct size quantification by MR imaging early after coronary artery occlusion in dogs. Radiology early after coron

5 Caputo GR, Sechtem V, Tscholakoff D, Higgins CB Measurement of myocardial infarct size at early and late Measurement of myocardial infarct size at early and late
time intervals using NMR imaging. An experimental time intervals using NMR imaging. An
study in dogs. Radiology 1987;149:237-43.

6 Been M, Smith MA, Ridgway JP, Brydon JWE, Douglas RHB, Kean DM, et al. Characterisation of acute myocardial infarction by gated magnetic resonance imaging. Lancet 1985;ii:348-50.

7 Tscholakoff D, Higgins CB, Sechtem U, Caputo G, Derugin N. MRI of reperfused myocardial infarct in dogs. AJR 1986;146:925-30.

8 Smith MA, Ridgway JP, Brydon JWE, Been M, Douglas RHB, Kean DM, et al. ECG gated T1 images of the heart. Phys Med Biol 1986;59:603-7.

9 Hutchinson JMS, Smith FW. In: Partain CL, James AE, Rollo FD, Price RR. Nuclear magnetic resonance (NMR) imaging. Philadelphia: Saunders, 231-49.

10 Jansen DE, Corbett JR, Wolfe CL, Lewis SE, Gabliani G, Filipchuk N, et al. Quantification of myocardial infarction: a comparison of single photon-emission computed a comparison of single photon-emission computed tomography with pyrophosphate to serial plasma MBcreatine

11 Muir AL, Hannan WJ, Sapru RP, Boardman AK, Wraith PK, Brash HM. The effects of isoprenaline, atropine and dobutamine on ventricular volume curves by radionuclid ventriculography. Clin Sci 1980;58:357-64.

12 Bland JM, Altmann DG. Statistical methods for assessing agreement between two methods of clinical measurement. Lancet 1986;i:307-10.

13 Dewhurst NG, Muir AL. Comparative prognostic value of radionuclide ventriculography at rest and during exercise in 100 patients after first myocardial infarction. Br Heart $J$ in 100 patients a

14 Page DL, Caulfield JB, Kaster JA, DeSanctis RW, Sanders CA. Myocardial changes associated with cardiogenic CA. Myocardial changes associated
shock. N Engl J Med 1971;285:133-7.

15 Alonso DR, Scheidt W, Post M, Killip T. Pathophysiology of cardiogenic shock. Quantification of myocardial necrosis: clinical, pathological, and electrocardiographic correlations. Circulation 1973;48:588-96.

16 Shell WE, Kjekshus JK, Sobel BE. Quantitative assessment of the extent of myocardial infarction in the conscious dog by means of analysis of serial changes in serum creatin phosphokinase activity. J Clin Invest 1971;50:2614-24.

17 Bleifeld WH, Hanrath P, Mathey D. Serial CPK determinators for evaluation of size and development of acute myocardial infarction. Circulation 1976;53(suppl 1): 108-11.

18 Kluge WF. Prognostic value of serum creatine phosphokinase levels in myocardial infarction. Northwest Med 1969;68:847-53.

19 I.S.A.M. Study Group. A prospective trial of intravenous streptokinase in acute myocardial infarction (I.S.A.M.): mortality, morbidity, and infarct size at 21 days. $N$ Engl $J$ Mortality, morbidity, and

20 Muller JE, Maroko PR, Braunwald E. Evaluation of precordial electrocardiographic mapping as a means of assessing changes in myocardial ischaemic injury. Circulation 1975; 52:16-27.

21 Murray RG, Peshock RM, Parkey RW, Bonte FJ, Willerson JT, Blomquist CG. ST iso-potential precordial surface maps in patients with acute myocardial infarction. J Electrocardiol 1979;12:55-64.

22 Corbett JR, Lewis M, Willerson JT, Nicod PH, Huxley RL, Simon $\mathrm{T}$, et al. Technetium-99m pyrophosphate imaging in acute myocardial infarction. Comparison of planar images with single-photon tomography with blood pool overlay. Circulation 1984;69:1120-8.

23 Lewis SE, Devous MD, Corbett JR, Izquierdo C, Nicod P, Wolfe CL, et al. Measurement of infarct size in acute canine myocardial infarction by single-photon emission computed tomography with technetium-99m pyrophosphate. Am J Cardiol 1984;54:193-9.

24 Buja LM, Parkey RW, Stokey EM, Bonte FJ, Willerson JT. Pathophysiology of technetium-99m pyrophosphate and Pathophysiology of technetium-99m pyrophosphate and thallium-201 scintigraphy of acute my
dogs. J Clin Invest 1976;57:1508-22.

25 Been M, Smith MA, Ridgway JP, Douglas RHB, de Bono DP, Best JJK, et al. Serial changes in the $T_{1}$ magnetic $\mathrm{DP}$, Best JJK, et al. Serial changes in the $T_{1}$ magnetic relaxation parameter after

26 Brown JJ, Higgins CB. Myocardial paramagnetic contrast agents for MR imaging. AJR 1988;151:865-72. 